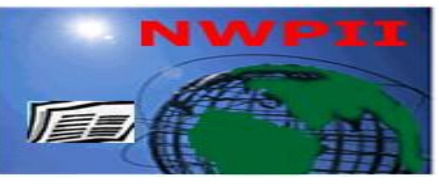

American Journal of Biomedical Sciences

ISSN: 1937-9080

nwpii.com/ajbms

\title{
Combinatorial Effect of Azadirachta Indica Seed Oil and Citrus Limon Peel Oil on Larvae and Adult of Mosquitoes
}

\author{
Abdulazeez A. ABUBAKAR PhD'ㄹ, Masturah M. GHALI B.Sc ${ }^{2}$, Ibrahim ABDULFATAH ${ }^{1}$ \\ ${ }^{1}$ Department of Medical Laboratory Science,University of Medical Sciences, Ondo-City,Nigeria. \\ Email:aabubakar@unimed.edu.ng Tel:+2348068039485 \\ ${ }^{1}$ Department of Medical Laboratory Science,University of Medical Sciences, Ondo-City Nigeria \\ Email:abdulfaibrahim@unimed.edu.ng Tel; +2348060118335 \\ ${ }^{2}$ Department of Microbiology,Kwara State University, Malete Kwara State \\ Email:abuazeez1962@gmail.com \\ "Corresponding Author \\ Dr. Abdulazeez Abubakar \\ Department of Medical Laboratory Science \\ University of Medical Sciences,Ondo-City \\ Nigeria. \\ Email:aabubakar@unimed.edu.ng \\ Tel: +2348068039485
}

Received:13 May 2019; | Revised:21 May 2019; $\mid$ Accepted:13 December 2019

\begin{abstract}
Emergence of resistant mosquitoes to Insecticide-treated nets and Indoor residual spraying is a threat to global malaria eradication program. The aim of the study is to investigate the combinatorial effect of Azadirachta indica seed oil and Citrus limon peel oil against mosquito larvae and adults. Fruits of Neem plant and peels of Unripe Lemon were obtained. The peels and seeds were separately washed, dried and blended into fine powder. Oil was extracted from each powder using absolute alcohol and separated using Soxhlet extractor. Each of the oils was diluted with absolute ethanol to obtain $20 \%, 30 \%, 40 \%, 50 \%$ and $60 \%$ concentrations. Equal quantities of the oils were mixed together and diluted with absolute ethanol to obtain fortified concentrations of $20 \%, 30 \%, 40 \%, 50 \%$ and $60 \%$. Five millilitres of each dilution was sprayed on adult mosquitoes and active larvae in a container of 100 millilitres of water and mortality rate calculated at 15 minutes interval for an hour. Complete mortality of adult mosquito (100\% mortality) was recorded at 60 minutes when $50 \%$ of lemon peel oil was administered while the same mortality rate was recorded with $60 \%$ concentration of Azadirachta indica seed oil at 60 minutes whereas the same optimum mortality was documented with $50 \%$ concentration of the combined oil extract. The lemon oil extract produced a promising mortality effect on mosquito larvae and adult mosquitoes hence could be explored for use as an alternative insecticide. Also, the findings revealed no synergistic or antagonistic effect between the two oil extracts.
\end{abstract}

Keywords: Azadirachta indica seed oil, Citrus limon peel oil, Mortality rate, Mosquito larvae and adults 


\section{Introduction}

Vector-borne diseases are responsible for about $17 \%$ of total infectious diseases worldwide with an annual death rate of about 700,000 [1]. Mosquito which transmits malaria parasites is also known to be a vector of many other disease agents. An estimated 219 million cases of malaria were reported worldwide with about 500,000 deaths in 2017 and Africa accounted for $92 \%$ of the cases ${ }^{[2]}$. Nigeria recorded $25 \%$ of the global cases and $19 \%$ of reported global death in $2017^{[3]}$. Children under 5 years old accounted for $61 \%$ of the deaths worldwide in $2017^{[3]}$.

Control of malaria depends largely on vector population control. Use of long-lasting Insecticidetreated nets and Indoor Residual Spraying (IRS) has shown a significant reduction in the incidence of malaria in Africa ${ }^{[4,5]}$. However, studies have been reports of resistance of mosquitoes to IRS [1,6] thereby threatening the global malaria eradication programme. These conditions necessitated the need for research on alternative agents that will be effective, non-hazardous, environmentally friendly and affordable.

Chemical compounds derived from different plants have been reported to have insecticidal activities against larvae and adult Anopheles mosquito $[7,8]$. Some of these chemicals act as toxicants and interfere with growth and all developmental stages of mosquitoes, whereas, others interfere with reproduction and olfactory receptors ${ }^{[9,10,11]}$.

The previous study revealed that Azadirachta indica (Neem) seed oil recorded a $100 \%$ mortality rate against mosquito within 72 hours after treatment with different concentrations of the oil extract ${ }^{[12,13]}$. Citrus limon (Lemon) peel oil has also been found as an effective agent in mosquito elimination ${ }^{[14]}$. Raguraman and Singh ${ }^{[15]}$ identified Citrus essential oil as important pesticides or insecticides.

However, the combinatorial effect of the two oils has not yet been documented. The aim of this study therefore is to investigate the combinatorial effects of the two oils on the mortality of mosquito larvae and adult mosquitoes

\section{Materials and Methods}

\subsection{Collection of Azadirachta indica seeds and unripe Citrus limon peels}

Fruits of Azadirachta indica plant were obtained from Malete, Elemere and Ilorin communities in Kwara State Nigeria. The peels and fleshy parts of the seeds were thoroughly washed away to expose the seed coats. The seed coats were sun-dried. After complete dryness, they were husked and the seeds were dried in the oven at $110 \mathrm{oC}$ for 5 hours. The dried seeds of Azadirachta indica were then ground into fine powder using a mechanical blender and the powder was kept in an airtight amber glass jar prior to extraction.

Unripe Citrus limon were procured in large quantities at Odo-Oba market in Ilorin Kwara state Nigeria, the peels were removed with a knife. These peels were oven-dried and ground using an electrical grinding machine into a fine powder before extraction.

\subsection{Preparation of Oil Extracts}

Each of the powders was poured into the thimble of separate Soxhlet extractor and cotton wool was used as the filter. Absolute ethanol was poured into the still pot as the solvent. The Still pot was placed in the water bath and the ethanol evaporates through condensation path of Soxhlet extractor where condensate of ethanol was allowed to drop on the powder in the thimble. The ethanolwashed down the oil, which then passed through the syphon and dropped onto the ethanol in the still pot in the water bath and the cycle continues until the extraction is completed (Reflux extraction). The extracted oil with the solvent was poured into a beaker and heated in the water bath until the ethanol was completely evaporated leaving just the oil.

\subsection{Dilution of Extracts}

Azadirachta indica seed oil and Citrus limon peel oil were separately diluted with absolute ethanol to obtain $20 \%, 30 \%, 40 \%, 50 \%$ and $60 \%$ concentrations respectively. Equal quantities of the oils were mixed together and diluted with absolute ethanol to obtain fortified concentrations of $20 \%$, $30 \%, 40 \%, 50 \%$ and $60 \%$. 


\subsection{Collection of Mosquito Larvae and Breeding of Adult Mosquito}

Larvae of the Mosquitoes were collected from stagnant water in tyres and broken containers around Kwara State University Student Hostels. Fifteen netted boxes measuring 6feet by 6 feet were specially constructed for breeding of adult mosquitoes. 100 mosquito larvae were kept in a small container of clean untreated water and kept inside each of the boxes. Each set of the box with the known mosquito larvae was used for each concentration of the oil extracts while the larvae were fed with ground crayfish. After 5-7 days, adult mosquito emerged and they were subsequently sprayed through the net while the box was tightly closed immediately and timing commenced to assess the mortality rate. The number of unhatched larvae was noted and subtracted from 100 to obtain the actual number of adult mosquito produced

\subsection{Application of the oil extracts on the mosquito larvae and adult}

Five millilitres of each dilution was sprayed on 100 active larvae in a container of $100 \mathrm{ml}$ of water and time taken immediately. The number of dead larvae was counted at an interval of 15 minutes, 30 minutes, 45 minutes, and 60 minutes. The mortality rate was calculated and recorded.

Similarly, $5 \mathrm{ml}$ of each dilution was sprayed on a known density of the adult mosquitoes in the box and time taken immediately. The number of dead adult mosquito was determined by using the touch light through the net when counting at the interval and to cross-check if the adult mosquitoes are still alive. The total number of larvae originally put in the container per box, minus the number of larvae not hatched is equal to number of adults that emerged. At an interval of 15 minutes, 30 minutes, 45 minutes and 60 minutes the number of dead mosquitoes was determined and the mortality rate calculated.

\subsection{Statistical Analyses}

Data obtained from the study were input and analysed with Statistical Package for Social Sciences software of computer (Version 23 SPSS Inc, USA). Chi-square test was used to assess the variations in the mortality rate of adult mosquito with respect to the concentration of Azadirachta indica seed oil, Lemon peel oil and combined oil extracts. The test for significance level was based on a $\mathrm{P}$ value less than 0.05 .

\section{Results}

Figure 1 shows the mortality rate of larvae at varying concentration of Azadirachta indica seed oil for 15, 30, 45 and 60 minutes. At 20\% concentration, after 15 minutes of application of the extract, the lowest mortality rate ( $20 \%$ mortality) was recorded. At the same concentration and after 30 Minutes the mortality rate remains unchanged till the end of the study. The highest mortality rate $(100 \%$ mortality) was recorded at $60 \%$ concentration after 60 minutes of application. Variations in the mortality rate of adult mosquito with respect to the concentration of Azadirachta indica seed oil extract is shown in Table 1. At $20 \%$ concentration, after 15 minutes of application, the lowest mortality rate $(20 \%$ mortality) was recorded whereas highest mortality rate $(100 \%$ mortality) was recorded at $60 \%$ concentration at 60 minutes. Statistical analysis by Chi-square depicted no significant in variations in the mortality rate of adult mosquito ( $\mathrm{X} 2=0.8764 ; \mathrm{P}>0.05)$ and mosquito larvae ( $\mathrm{X} 2=0.7886 ; \mathrm{P}>0.05)$ with respect to the concentration of Azadirachta indica seed oil

Figure 2 depicts the variations in the mortality rate of mosquito larvae with respect to the concentration of Lemon peel oil extract. At 20\% concentration at 15 minutes time, the lowest mortality rate $(40 \%$ mortality) was recorded whereas the highest mortality rate $(100 \%$ mortality) was recorded at $50 \%$ concentration at 60 minutes time

Although there are a varying number of adult mosquitoes that emerge from 100 larvae bred in the box, the mortality rate was based on the total number of larvae originally put in the container per box, minus the number of unhatched larvae. The mortality rate of mosquitoes at different concentration of Citrus lemon peel oil at 15, 30, 45 and 60 minutes was represented in Table 2. The lowest mortality rate (38\% mortality) was recorded at $20 \%$ concentration after 15 minutes and the mortality rate increases as the concentration and time increase. Chi-square analysis also revealed an insignificant difference in the mortality rate of adult 
mosquito ( $\mathrm{X} 2=1.1704 ; \mathrm{P}>0.05)$ and mosquito larvae $(\mathrm{X} 2=0.9387 ; \mathrm{P}>0.05)$ with respect to the concentration of Citrus lemon peel oil

Figure 3 depicts the mortality rate of the larvae at varying concentrations of the combined oil after applying them for 15, 30, 45 and 60 minutes. At $20 \%$ concentration for 15 minutes, the lowest mortality rate was recorded ( $40 \%$ mortality) while the highest mortality rate $(100 \%$ mortality) was recorded at $50 \%$ concentration, 45 minutes after application.
The rate of death of the adult mosquito at varying concentrations of the combined oil at 15, 30, 45 and 60minutes is shown in Table 3. The lowest mortality rate ( $44 \%$ mortality) was observed at $20 \%$ concentration while $50 \%$ concentration recorded the highest mortality rate. Chi-square analysis showed that variations in the mortality rate of adult mosquito $(\mathrm{X} 2=0.9705 ; \mathrm{P}>0.05)$ and mosquito larvae $(\mathrm{X} 2=0.9387 ; \quad \mathrm{P}>0.05)$ are statistically insignificant with respect to the concentration of combined oil extract.

Table 1: Variations in the mortality rate of adult mosquito with respect to the concentration of Azadirachta indica seed oil extract

\begin{tabular}{cccccc}
\hline TIME (MINUTES) & \multicolumn{3}{c}{ CONCENTRATION } & $\mathbf{5 0 \%}$ & $\mathbf{6 0 \%}$ \\
\hline 15 & $\mathbf{2 0 \%}$ & $\mathbf{3 0 \%}$ & $\mathbf{4 0 \%}$ & 46 & 52 \\
30 & 20 & 28 & 40 & 56 & 68 \\
45 & 46 & 48 & 52 & 68 & 82 \\
60 & 54 & 54 & 66 & 98 & 100
\end{tabular}

$\mathrm{X}^{2}=0.8764$

Table 2: Variations in the mortality rate of adult mosquito with respect to the concentration of Lemon peel oil extract

TIME (MINUTES)

CONCENTRATION

\begin{tabular}{cccccc} 
& $\mathbf{2 0 \%}$ & $\mathbf{3 0 \%}$ & $\mathbf{4 0 \%}$ & $\mathbf{5 0 \%}$ & $\mathbf{6 0 \%}$ \\
\hline 15 & 38 & 44 & 46 & 54 & 58 \\
30 & 54 & 56 & 56 & 68 & 72 \\
45 & 56 & 64 & 70 & 86 & 84 \\
60 & 70 & 76 & 82 & 100 & 100
\end{tabular}

$\mathrm{X}^{2}=1.1704$

Table 3: Variations in the mortality rate of adult mosquito with respect to the concentration of combined oil TIME (MINUTES) CONCENTRATION extract

\begin{tabular}{cccccc} 
& $\mathbf{2 0 \%}$ & $\mathbf{3 0 \%}$ & $\mathbf{4 0 \%}$ & $\mathbf{5 0 \%}$ & $\mathbf{6 0 \%}$ \\
\hline 15 & 44 & 50 & 46 & 60 & 68 \\
30 & 52 & 58 & 56 & 72 & 86 \\
45 & 62 & 70 & 96 & 100 & 100 \\
60 & 84 & 88 & 94 & 100 \\
\hline
\end{tabular}

$\mathrm{X}^{2}=0.9705$ 


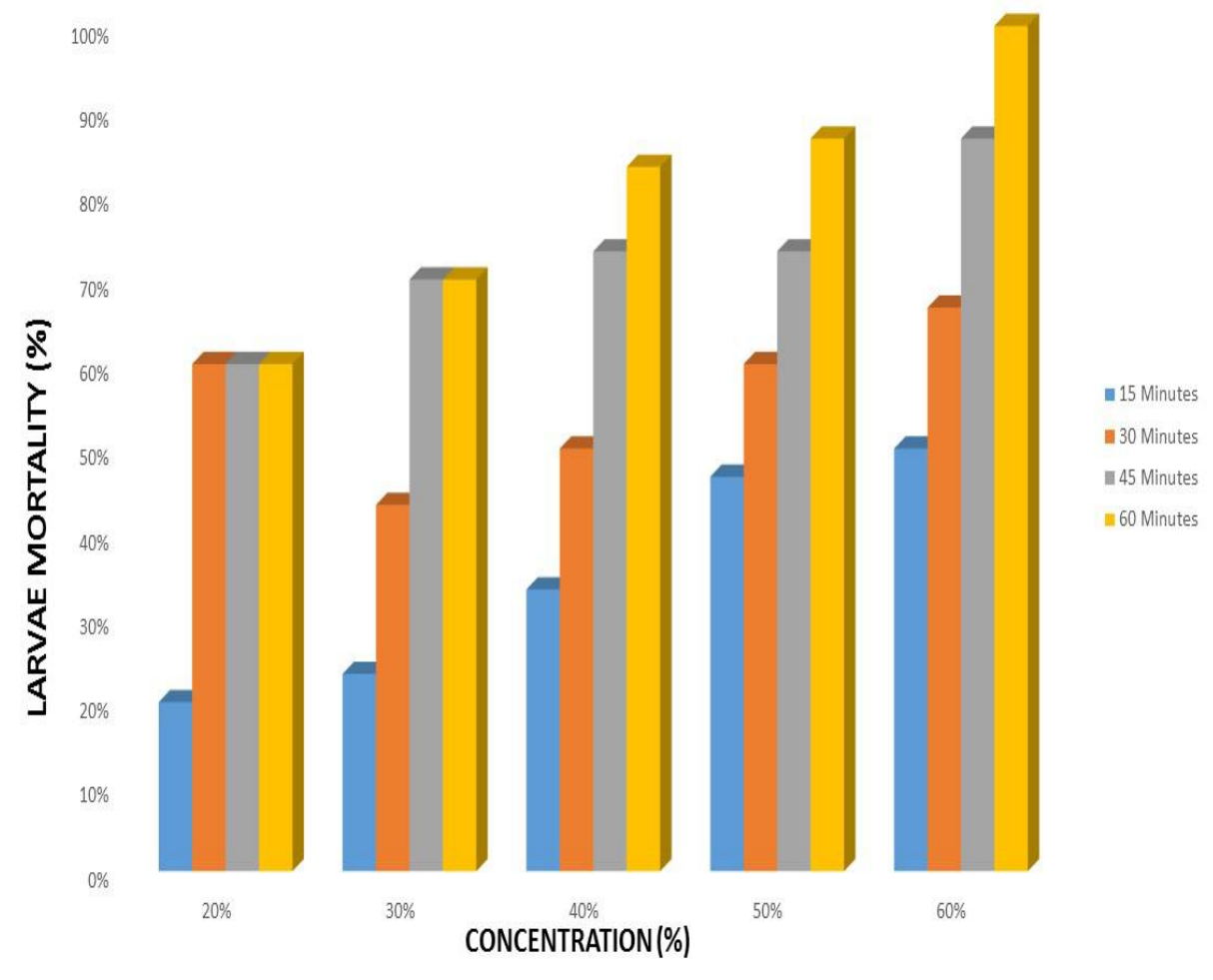

Figure 1: Variations in the mortality rate of mosquito larvae with respect to the concentration of Azadirachta indica seed oil extract

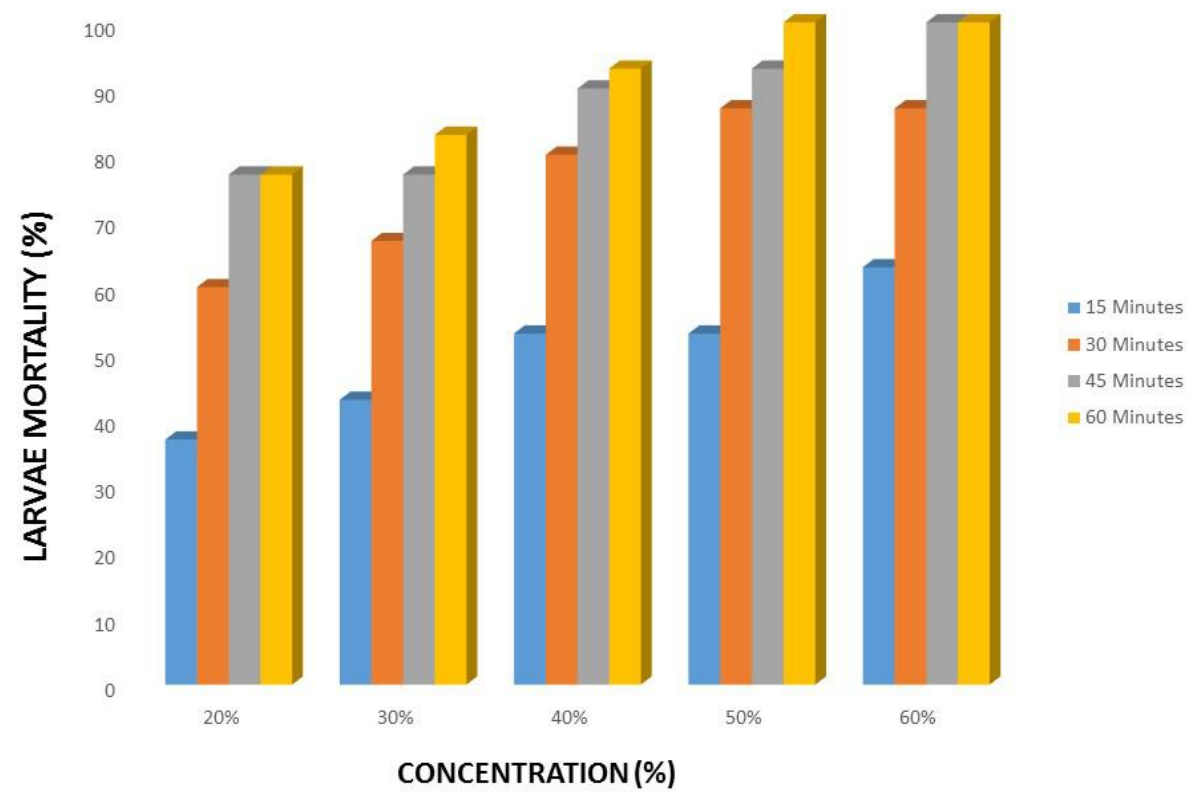

Figure 2: Variations in the mortality rate of mosquito larvae with respect to the concentration of Lemon peel oil extract 


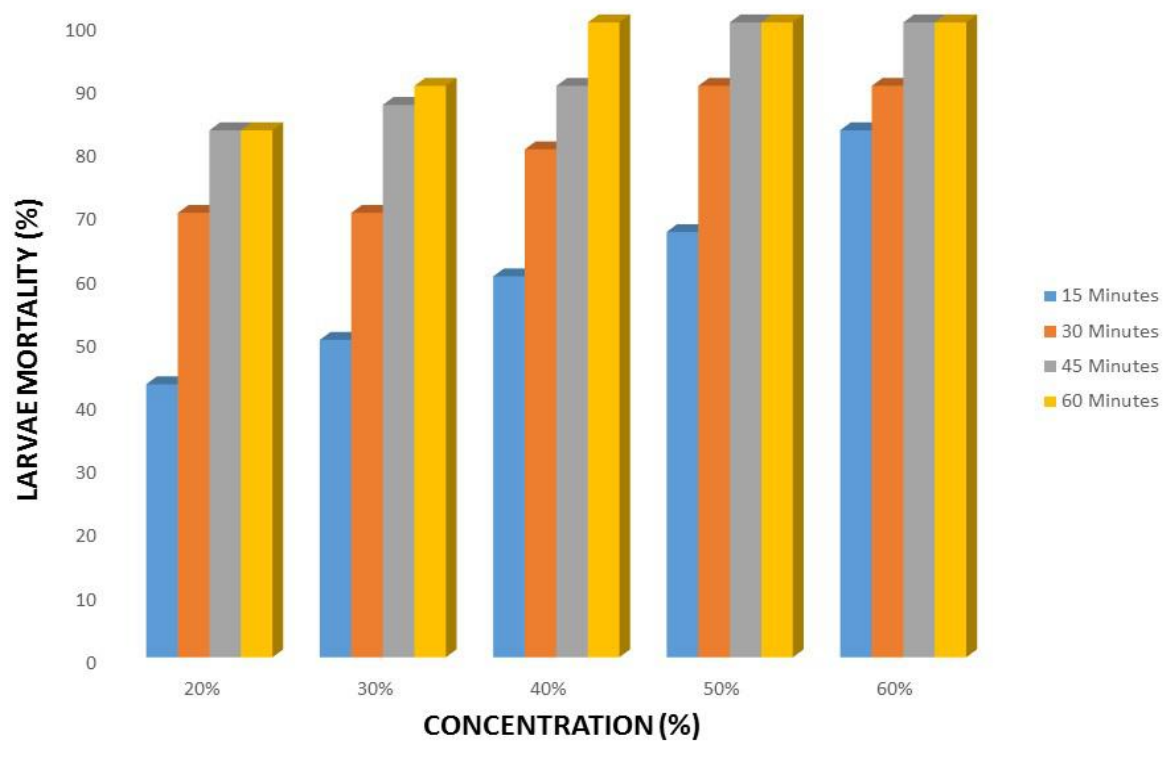

Figure 3: Variations in the mortality rate of mosquito larvae with respect to the concentration of combined oil extract

\section{Discussion}

Our finding in this study shows that mortality effect of the seed oil of Azadirachta indica at a concentration of $20 \%$ on mosquito larvae did not increase appreciably with an increase in time of exposure. However, at a concentration of $60 \%$, complete mortality of mosquito larvae (100\% mortality) was recorded 60 minutes after exposure to the seed oil extract. The finding is in line with a similar study by Shivakumar and Kataria ${ }^{[12]}$ where the mortality rate of $100 \%$ was produced 72 hours after treatment of Anopheles stephensi larvae with Neem extract. In another study by Okumu, Knols and Fillinger there was about $25 \%$ reduction in the adult longevity of larvae that were exposed to a lower concentration of Neem oil formulation. The mortality effect on larvae was linked to disruption of the metamorphosis of the larvae ${ }^{[16]}$. The effect on larvae is important to reduce metamorphosis into adult mosquitoes which is crucial for lowering a vector's disease transmission potential.

Also, as the time of exposure of Azadirachta indica seed oil increases, the mortality rate of adult mosquito also increases considerably with an increase in concentration. At $60 \%$ concentration for 60 minutes, complete mortality (100\% mortality) was recorded. Previous research by Hati et al, [17] showed that neem (Azadirachta indica) seed oil performed optimally as an excellent mosquito repellent when smeared on the exposed part of the body and also has the insecticidal ability ${ }^{[18]}$. Similarly, Alyssa, ${ }^{[19]}$ reported repellent action of the neem against mosquito at varying concentrations. Research conducted in Nigeria [20] however reported that extract of the seed kernel of Azadirachta indica showed effective repellent activity against female Anopheles mosquito species. The active compounds found in extracts of Azadirachta indica are Azadirachtin and Nimbim which have been reported to have toxic effects on insects ${ }^{[21]}$. The Azadirachtin displays neurotoxic effects on insects by altering the functions of the central nervous system (CNS) through inhibiting the excitatory cholinergic transmission and blocking the calcium channels partly ${ }^{[22,23,24]}$.

Effect of $60 \%$ concentration of Citrus limon peel oil on mosquito larvae depicts an optimum mortality rate $(100 \%$ mortality) after 60 minutes exposure while the effect of the Citrus limon peel oil at $20 \%$ concentration was $77 \%$.

Our present findings also revealed there was an increase in mortality rate of mosquitoes as the time of exposure and concentration of the oil increased 
with the maximum mortality rate recorded at $50 \%$ and $60 \%$ concentrations. Similarly, this research showed maximum larvicidal mortality at 50\% concentrations and $60 \%$ concentrations of Citrus limon peel oil which is in agreement with mortality rate recorded in studies of Mansour and his Associates [9] where optimum mortality rate was also observed against mosquito larvae and adults. In another study [25], Lemon peel oil was found to be the most toxic followed by Orange and Sour Orange peel oils against the larvae of Culex quinuefasciatus.

In a previous study, the repellent activity of the volatile phytochemical extracts of citrus fruits was reported and found to exhibit repellent activities at varying concentrations ${ }^{[26]}$.

Comparing the efficacy of the individual oils with the combined concentration of the oils, the combination shows a relatively higher degree of efficacy than Azadirachta indica seed oil but performs almost equally as Citrus limon peel oil. This result disagrees with the findings of Abubakar et al., ${ }^{[27]}$ where they observed a synergistic effect of two fortified extracts on Plasmodium species, although, the authors employed Bryophillim pinnatum and Aloe barbadensis. Therefore our finding discourages the need to combine citrus peel oil and Azadirachta indica seed oil for use as a mosquitocidal agent.

\section{Conclusion}

Comparing the mortality effects of both oils on mosquito larvae and adults, lemon oil extract appears to be more efficacious and may serve as a promising and better alternative than neem oil. Also, the findings revealed that there was no synergistic or antagonistic effect of one oil extract on the other

We, therefore, recommend lemon oil extract as a replacement of some synthetic, ineffective and hazardous environmentally unsafe insecticides.

\section{References}

1 World Health Organizations (WHO). Vectorborne diseases. 2017. [Accessed 24 January, 2019]

2 Yeka A, Gasasira A, Mpimbaza A, Achan J, Nankabirwa J, Nsobya S, Staedke SG, Donnelly MJ, Wabwire-Mangen F, Talisuna A,
Dorsey G, Kamya MR, Rosenthal PJ. Malaria in Uganda: challenges to control on the long road to elimination: I. Epidemiology and current control efforts. Acta Trop 2012; 121(3): 184-195 [PMID: 21420377 DOI: 10.1016/j.actatropica.2011.03.004]

3 World Health Organization (WHO). This year's Malaria Report at a glance. World Malaria Report. 2018. [Accessed 18 February, 2019]

4 Bhatt S, Weiss DJ, Cameron E, Bisanzio D, Mappin B, Dalrymple U, Battle K, Moyes CL, Henry A, Eckhoff PA, Wenger EA, Briet O, Penny MA, Smith TA, Bennett A, Yukich J, Eisele TP, Griffin JT, Fergus CA, Lynch M, Lindgren F, Cohen JM, Murray CLJ, Smith DL, Hay SI, Cibulskis RE, Gething PW. The effect of malaria control on Plasmodium falciparum in Africa between 2000 and 2015. Nature 2015; 526(7572): 207-211 DOI: 10.1038/nature 15535

5 Ulrich JN, Naranjo DP, Alimi TO, Muller GC, Beier JC. How much vector control is needed to achieve malaria elimination? Trends Parasitol 2013; 29(3): 104-109 DOI: 10.1016/j.pt.2013.01.002

6 Ranson H, Rossiter L, Ortelli F, Jensen B, Wang X, Roth CW, Collins FH, Hemingway J. Identification of a novel class of insect glutathione S-transferases involved in resistance to DDT in the malaria vector Anopheles gambiae. Biochem $\boldsymbol{J}$ 2001; 359(Pt 2): 295-304 DOI: 10.1042/0264-6021:3590295

7 Zia, K.; Hafeez, F.; Ashfaq, M.; Akram, W.; Bashir, M. H.; Khan, H. A. A. Larvicidal action off our indigenous plant extracts against dengue vector aedes aegypti. Pak Entomol 2012, 34(1): 93-97

8 Ghosh A, Chowdhury N, Chandra G. Plant extracts as potential mosquito larvicides. Indian J Med Res 2012; 135(5): 581-598 [PMID: 22771587 PMCID: PMC3401688]

9 Mansour, A. S.; El-Sharkawy, Z. A.; Ali, R. A. Botanical Biocides.12. Botanical biocides. 12. Mosquitocidal Activity of Citrus Peel Oils With Respect To Their Limonene Content. Egyptian Journal of Nature Toxins. 2004, 1: 111-134

10 Sukumar, K.; Perich, M.J.;Boobar, L. R. Botanical derivatives in mosquito control: A 
review. Am Mosq Control Assoc 1991, 7: 210237

11 Nyasembe VO, Torto B. Volatile phytochemicals as mosquito semiochemicals. Phytochem Lett 2014; 8: 196-201 DOI: 10.1016/j.phytol.2013.10.003

12 Shivakumar, M. S.; Kataria, R. Comparative efficacy of Azadirachtin on larval population of Culex quinquefasciatus, Anopheles stephensi and Aedes aegypti (Diptera: Culicidae) in Gujaret, India. Int J Pharm Bio Sci 2011, 2: 41-47.

13 Dua VK, Pandey AC, Raghavendra K, Gupta A, Sharma T, Dash AP. Larvicidal activity of neem oil (Azadirachta indica) formulation against mosquitoes. Malar $\boldsymbol{J}$ 2009; 8: 124 DOI: $\underline{10.1186 / 1475-2875-8-124}$

14 Dosoky NS, Setzer WN. Biological Activities and Safety of Citrus spp. Essential Oils. Int J Mol Sci 2018; 19(7) DOI: $\underline{\text { 10.3390/ijms19071966 }}$

15 Raguraman, S.;Singh, D. Biopotentials of Azadirachta indica and Citrus deodora oils on Callosobruchus sinensis. J Pharmacol 1997, 35:344-348

16 Schmutterer, H. The Neem Tree: Cource of Unique natural Products for Integrated Pest management, Medicine, Industry and Other Purposes. VCH, Weinheim Germany. 199; 1696.

17 Hati, A.K.;Bhowmik, K.;Banerjee, A. Repellent action of neem (Azadirachta indica) seed oilagainst Aedes aegypti mosquitoes. Indian $J$ Dermatol 1995, 40(4): 155-158

18 Mordue, L. Azadirachtin: an Update. J Insect Physiol 1993, 39: 903-924

19 Alyssa,M.T.Neem spray as mosquito repellent. Science Res 2010, 11-12.
20 Wannang, N. N.; Ajayi, V. F.; Ior, L. D.; Dapar, L. M. P.; Okwori, V. A.; Ohemu, T. Mosquito Repellant Property of Azadirachta Indica Extract (Fruit Bark and Seed Kernel) Scientific Research Journal (SCIRJ), Volume III, Issue VI, June 2015

21 Mordue, A. J.; Nisbet, A. J. Azadirachta form the neem tree Azadirachta indica its actions against insects. Am Soc Entomol Bras 2008, 29: 615-632.

22 Qiao, J.; Zou, X.; Lai, D.; Yan, Y.; Wang, Q.; Li, W.; Deng, S.; Xu, H.; Gu, H. Azadirachtin blocks the calcium channel and modulates the cholinergic miniature synaptic current in the central nervous system of Drosophila. Pest Manag Sci 2014, 70: 1041 -1047.

23 Senthil, N. S.; Young, C. M.; Yul, S. H.; Hoon, P. C.; Kalaivani, K.; Duk, K. J. Effect of azadirachtin on acetylcholinesterase (AChE) activity and histology of the brown planthopper Nilaparvata lugens (Stal). Ecotoxicol Environ Saf 2008, 70: 244-250.

24 Hertel, W.;Muller, P. J. Physiological effects of the natural products quassin, cinnamaldehyde and azadirachtin on Periplaneta americana (L.). J Appl Entomol 2006, 130: 323-328

25 Mwaiko, G. L. Citrus peel extracts as mosquito larvae insecticides. East Afric Med J 1992, 69(4): 223-226

26 Effiom, O. E.; Avoaja, D. A.; Ohaeri, C. C. Mosquito Repellent Activity of Phytochemical Extracts from Peels of Citrus Fruit Species. Global J Sci Frontier Res 2012, 12(1)

27 Abubakar, A. A.; Kolawole, D. O.; Babatunde, S. K.; Sunday, O.; Ameen, N. Investigation of mosquitocidal activity of a combined extract of Bryophyllum pinnatum and Aloe barbadensis leaves. Agric Biol J North Amer 2014; 5 (3): 118-122 